\title{
Touching a Rubber Hand: Feeling of Body Ownership Is Associated with Activity in Multisensory Brain Areas
}

\author{
H. Henrik Ehrsson, ${ }^{1}$ Nicholas P. Holmes, ${ }^{2}$ and Richard E. Passingham ${ }^{1,2}$ \\ ${ }^{1}$ Wellcome Department of Cognitive Neurology, Institute of Neurology, London WC1N 3BG, United Kingdom, and ${ }^{2}$ Department of Experimental \\ Psychology, University of Oxford, Oxford OX1 3UD, United Kingdom
}

In the "rubber-hand illusion," the sight of brushing of a rubber hand at the same time as brushing of the person's own hidden hand is sufficient to produce a feeling of ownership of the fake hand. We shown previously that this illusion is associated with activity in the multisensory areas, most notably the ventral premotor cortex (Ehrsson et al., 2004). However, it remains to be demonstrated that this illusion does not simply reflect the dominant role of vision and that the premotor activity does not reflect a visual representation of an object near the hand. To address these issues, we introduce a somatic rubber-hand illusion. The experimenter moved the blindfolded participant's left index finger so that it touched the fake hand, and simultaneously, he touched the participant's real right hand, synchronizing the touches as perfectly as possible. After $\sim 9.7 \mathrm{~s}$, this stimulation elicited an illusion that one was touching one's own hand. We scanned brain activity during this illusion and two control conditions, using functional magnetic resonance imaging. Activity in the ventral premotor cortices, intraparietal cortices, and the cerebellum was associated with the illusion of touching one's own hand. Furthermore, the rated strength of the illusion correlated with the degree of premotor and cerebellar activity. This finding suggests that the activity in these areas reflects the detection of congruent multisensory signals from one's own body, rather than of visual representations. We propose that this could be the mechanism for the feeling of body ownership.

Key words: premotor cortex; posterior parietal cortex; cerebellum; rubber-hand illusion; functional magnetic resonance imaging; body image; self recognition

\section{Introduction}

When we touch or look at our hands, we immediately feel that they are part of our own body. This experience of the body as part of the self is a fundamental aspect of self-consciousness. It has been suggested that self-attribution of body parts is mediated by multisensory perceptual correlations (Bahrick and Watson, 1985; Mitchell, 1997; Botvinick and Cohen, 1998; Rochat, 1998; van den Bos and Jeannerod, 2002). For example, the attribution of a seen hand to the self would depend on a match between the somatic and visual signals from the hand. The recently discovered "rubber-hand illusion" supports this view by demonstrating that synchronous brushing of a visible rubber hand and the person's own hand, which is hidden from view, produces a feeling of ownership of the fake hand (Botvinick and Cohen, 1998).

We recently used functional magnetic resonance imaging (fMRI) to scan participants' brain activity while they perceived this illusion (Ehrsson et al., 2004). We found increases in activity in the bilateral ventral premotor cortex, the left intraparietal cortex, and the bilateral cerebellum during the rubber-hand illusion.

Received March 1, 2005; revised 0ct. 6, 2005; accepted 0ct. 6, 2005.

H.H.E. was supported by a postdoctoral stipend from the Human Frontier Science Program. N.P.H. was supported by Wellcome Studentship 065696/Z/01/A from The Wellcome Trust.

Correspondence should be addressed to $\mathrm{H}$. Henrik Ehrsson, Institute of Neurology, Wellcome Department of Imaging Neuroscience, 12 Queen Square, London WC1 N3BG, UK. E-mail: h.ehrsson@fil.ion.ucl.ac.uk. DOl:10.1523/JNEUROSCI.0800-05.2005

Copyright $\odot 2005$ Society for Neuroscience 0270-6474/05/2510564-10\$15.00/0
Importantly, bilateral activity in the premotor cortex seemed to reflect the feeling of ownership of the hand and this activity correlated with the strength of the perceived illusion. We pointed out that the premotor cortex receives both visual and somatic information (Rizzolatti et al., 1981a,b, 1998; Graziano, 1999; Lloyd et al., 2003) and argued that it is the correlation between these two sources of information that underlies the illusion of ownership.

In commenting on our findings, Botvinick (2004) pointed to evidence that cells in the premotor cortex respond both when a specific area of the body is touched and when an object is seen approaching that area (Rizzolatti et al., 1981a,b; Gentilucci et al., 1983; Fogassi et al., 1992; Graziano et al., 1994). These cells code visual inputs in a body-centered reference frame (Graziano et al., 1994, 1997a). The suggestion is that the premotor activity in our study may reflect a shift in hand-centered visual receptive fields, so that they became aligned with the artificial hand. If so, this activity may reflect a response to an object (the brush) in peripersonal space rather than the self-attribution of the artificial hand.

It is clearly important to find out whether an illusory feeling of ownership can be induced in the absence of visual input (i.e., without a change in visual receptive fields). In the present psychophysical and fMRI experiments, we demonstrate that this can also be achieved by synchronous tactile and proprioceptive stimulation in the absence of any visual input, when the participants' eyes are closed. We scanned blindfolded participants during a somatic version of the rubber-hand illusion, when they were 
touching a rubber hand but feeling as if they were touching their own hand (for a summary of the experimental design, see Fig. 1). We tested the hypothesis that activity in the ventral premotor cortex (Ehrsson et al., 2004), and perhaps in the intraparietal cortex and cerebellum, would reflect the feeling of ownership of the hand rather than a visual representation of the hand being brushed.

\section{Materials and Methods Psychophysical experiments}

\section{Participants}

Thirty-two right-handed healthy participants (15 females; aged 20-46 years) took part in the psychophysical experiment. All participants had given their written consent, and the study was approved by the joint National Hospital for Neurology and Neurosurgery/Institute of Neurology Ethics Committee.

\section{Procedures: the illusion stimulation}

The participants were blindfolded and seated with their arms resting on a table in a pronated position (palms down) (see Fig. 1, top). A rubber, life-size prosthesis of a male or female right hand (gender matched) was placed on the table between the participant's hands $(15 \mathrm{~cm}$ to the left of the participant's right hand). The participants, the experimenter, and the rubber hand all wore identical plastic surgical gloves to make the tactile surfaces of the two hands as similar as possible to each other. The experimenter moved the participant's left index finger so that it touched the right rubber hand on the knuckle of the index finger (metacarpophalangeal joint). At the same time, the experimenter touched the knuckle of the participant's right hand, synchronizing the touches on the two hands as closely as possible. This stimulation corresponds to the illusion condition as defined in the fMRI experiment described below. The stimulation lasted for $60 \mathrm{~s}$ (experiment 1 ) or $30 \mathrm{~s}$ (experiments 2 and 3 ).

\section{Experiment 1: rubber-hand illusion questionnaire}

After a $60 \mathrm{~s}$ stimulation period, as described in the previous paragraph (illusion), the participants completed a "rubber-hand illusion questionnaire." Five questions, based on those used by Botvinick and Cohen (1998), were designed that required a rating of the strength of agreement or disagreement with five perceptual effects. The first statement, which was designed to correspond to the illusion, was (1) "I felt as if I was touching my right hand with my left index finger" (this is abbreviated as "touching own hand") (see Figs. 2, 3). The four other statements, which were unrelated to the illusion, served as control statements for suggestibility: (2) "more than one" (they felt that they had more than one righthand hand), (3) "larger hand" (that is, their right hand felt larger than normal), (4) "moving hand" (they experienced that their right hand was moving), and (5) "not feel hand" (they could not feel their own right hand). The participants used a seven-point visual analog scale to rate the extent to which these statements did or did not apply. On this scale, 1 meant "absolutely certain that it did not apply," 4 meant "uncertain whether or not it applied," and 7 meant "absolutely certain that it applied."

\section{Experiment 2: quantifying vividness and continuance of illusion} The illusion condition was tested, but this time the participants were instructed to verbally report when the illusion started, and then stimulation was continued for $30 \mathrm{~s}$ after this response. We asked the participants to rate the vividness and continuance (persistence) of the illusion after the reported illusion onset, using a visual analog scale (from 0 to 9). The vividness was described to participants as "how lifelike and realistic was the feeling that they were touching their own hand." The continuance score reflected the proportion of time during which the illusion was experienced during the stimulation trial.

\section{Experiment 3: poststimulation pointing responses}

In the next experiment, we examined whether the illusion was associated with a drift in the felt position of the hand, because this has been reported for the visual rubber-hand illusion (Botvinick and Cohen, 1998; Tsakiris and Haggard, 2005). For periods of 30 s, we exposed 28 of the 32 participants to the illusion condition (synchronous) and a control condition, presented three times each in a counterbalanced order across participants. The control stimulation was asynchronous touches applied to the rubber hand and the real hand, which were shown in pilot experiments not to elicit the illusion (asynchronous). Immediately before and after the stimulation trials, the blindfolded participants were required to point to the tip of their right index finger using their left index finger as follows. The rubber hand was removed from the table, but the participants kept their right hand in position, and then stretched their left $\operatorname{arm} 45^{\circ}$ out and to the left from the body's parasagittal plane. The participants then moved their left index finger in a straight line to the felt position of the tip of their right index finger, making a single brisk movement. A scale mounted on the table was used to measure the distance between the right index finger and the left index finger after each pointing movement. The pointing error was calculated as the distance between the two index fingers after the stimulation period minus the distance between the index fingers before the stimulation period. Pointing errors were analyzed with a one-tailed paired $t$ test, with the prediction that pointing errors should be greater in the illusion condition than in the control condition (asynchronous).

\section{Brain-scanning experiment}

\section{Participants and additional psychophysics}

Fifteen participants (eight females; aged 21-40) were selected to take part in the brain-scanning experiment. These were randomly drawn from a group of 25 people who experienced the illusion in the initial psychophysical testing. For these participants, we quantified the illusion for the three experimental conditions in a test session outside the scanner (illusion, asynchronous, and incongruent) (for details, see below, Experimental design). Each condition was tested for $60 \mathrm{~s}$ with the order of the conditions being counterbalanced across participants. After each condition, the participants completed the rubber-hand illusion questionnaire as described above (for results, see Fig. 3).

\section{Experimental design}

While the brain scans were being performed, the participants rested comfortably in a supine position on the bed in the magnetic resonance imaging (MRI) scanner. All participants wore headphones to reduce noise and to receive auditory cues. They were blindfolded and asked to keep their eyes closed. To reduce potential head movements, we fixed the position of the head using foam pads. As in the psychophysical experiments described above, the participant and the experimenter both wore the same plastic surgical gloves on both hands.

The participant's right and left arms rested on a small table, which was placed over their stomach (Fig. 1, bottom). The participants extended their arms in parallel with their body, and foam pads were used to support their arms in a relaxed position. The rubber hand was placed between the participant's two hands on the table, aligned in parallel with and $15 \mathrm{~cm}$ to the left of the participant's own right hand. The rubber hand wore the same plastic glove as the participant and the experimenter. A small stiff plastic brush $(2 \times 3 \mathrm{~cm})$ was attached to the lateral side of the index finger of the rubber hand. The participant's left hand was placed so that the tip of the index finger could touch either the knuckle of the rubber hand or the brush beside it. The experimenter stood on the participant's left side and grasped the participant's left index finger with his left hand, and placed his right index finger just above the participant's right hand. Thus, the experimenter could move the participant's left index finger passively so that it touched the rubber hand (or the brush), and, at the same time, he could touch the participant's right hand on the corresponding site. The participants were instructed to relax completely, and in a short test session before the scan started, the participants had been trained to relax their left index finger as the experimenter moved it.

In the brain imaging experiment, there were three experimental conditions and one rest condition serving as the baseline. The conditions are described below.

Illusion. The experimenter moved the participants left index finger so 

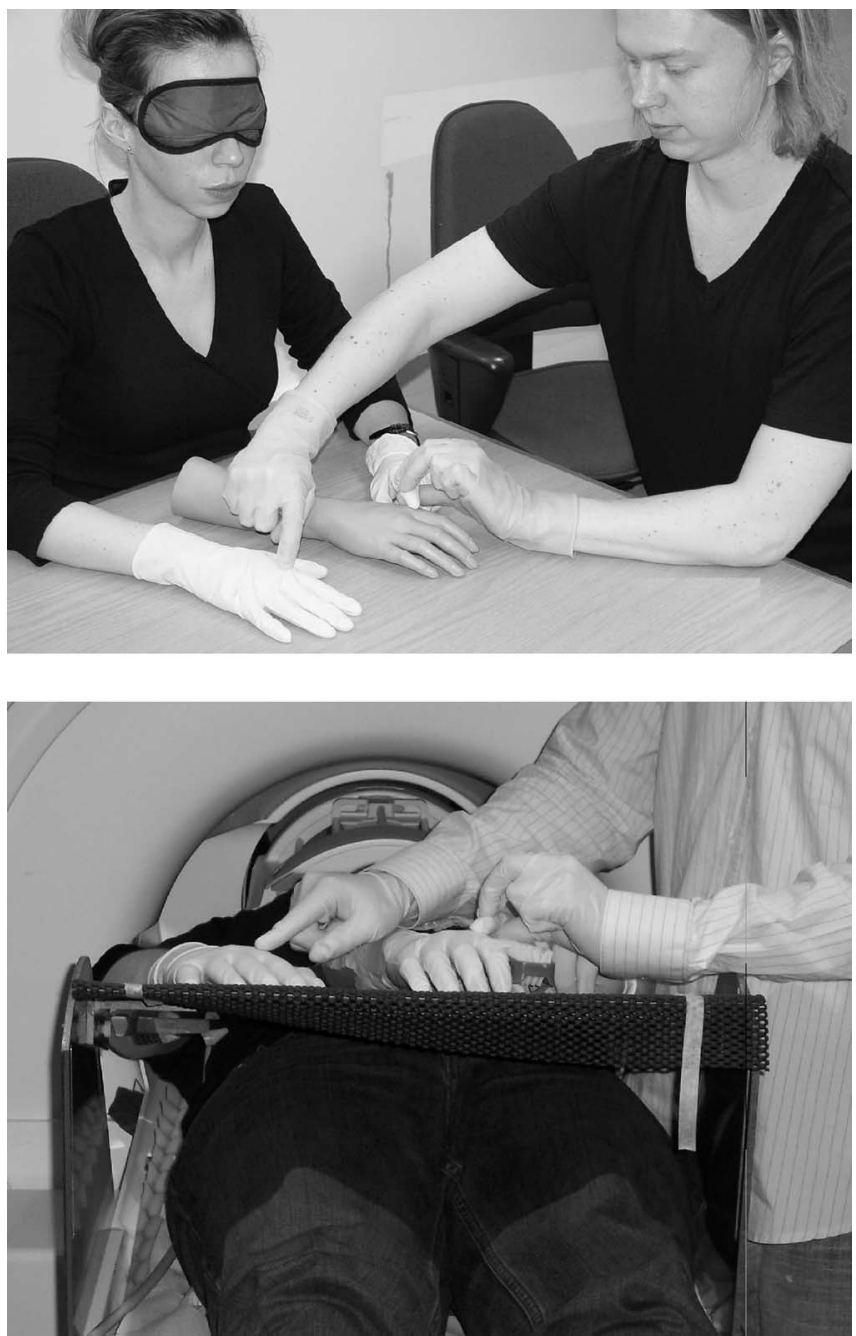

Figure 1. The somatic rubber-hand illusion. The experimenter moved each participant's left index finger so that it touched the right rubber hand on the knuckle of the index finger, and at the same time, the experimenter touched the participant's right index finger on the knuckle, synchronizing the touches on the two hands as closely as possible. Tapping movements were applied to the two hands at $1 \mathrm{~Hz}$, which, after a period of $\sim 10 \mathrm{~s}$, elicited an illusion that the participants were touching their own hand (illusion). The illusion was not elicited in the contro conditions when asynchronous touches were applied (asynchronous), or if the participants were touching a brush rather than the rubber hand (incongruent). The top panel illustrates the setup with a sitting participant, as used in our initial psychophysical experiment. The bottom panel shows the setup used in the brain-scanning experiment. In the brain scan experiments (bottom), a small brush was attached to the lateral side of the rubber hand to be used in the incongruent control condition.

that it touched the rubber hand and simultaneously touched the participant's right hand, synchronizing the touches as exactly as possible. This stimulation elicits the illusion that one is touching one's own hand.

Asynchronous. As in the previous condition, the experimenter moved the participant's left finger so that it touched the rubber hand and touched the participant's right hand. However, the touches on the two hands were alternated (i.e., they were asynchronous). Typically, this stimulation did not elicit the illusion of self-touch during pilot testing. Instead, the participants felt both that they are touching an external object and that someone else is touching their right hand.

Incongruent. The experimenter moved the participants left index finger so that it touched the brush and simultaneously touched the participant's right hand, synchronizing touches. Because the texture of the brush was very different compared with the rubber hand and the participant's own hand, no illusion of self-touch was typically elicited.
Rest. The participants lay at rest with their eyes closed and the right hand in a relaxed position without touching the rubber hand.

The movements of the participant's left index finger were small and brisk with a mean frequency of $1 \mathrm{~Hz}$. Likewise, touches to the participant's right hand were applied with a mean frequency of $1 \mathrm{~Hz}$. The timing of the touches was irregular (i.e., not isochronous) because pilot experiments had shown that this caused a more vivid illusion than regular tapping (Armel and Ramachandran, 2003). The experimenter listened to a metronome, which provided a base frequency of $1 \mathrm{~Hz}$, and then varied the interval between the touches from 0.5 to $1.5 \mathrm{~s}$ in a pseudorandom manner. Only the experimenter could hear the metronome. Two small potentiometers attached on the participant's left index finger and the experimenter's right index finger registered the number of touches and the amplitude of the movements. Importantly, the number of movements of the left index finger and the number of touches on the right hand were identical in all conditions. Likewise, the amplitude of the passive left-finger movements was matched.

Each condition lasted $42 \mathrm{~s}$. To indicate the onset of the illusion in the illusion condition, the participants were instructed to press a key pad with the left foot in a relaxed manner when they first started to feel the illusion that the hand they were touching was their own. When they pressed the key, they heard a brief tone in the earphones to match the tone presented in the other conditions (see below). The reported onset of the illusion was $9.7 \pm 5.3 \mathrm{~s}$ (mean \pm SD across participants; mean within participant SD was $3.8 \mathrm{~s}$ ) after the beginning of the trial, ensuring an average of over $32 \mathrm{~s}$ of stimulation per trial. In the asynchronous and incongruent conditions, the participants were required to make a key press with their foot when they heard the tone. Thus, the foot response was matched in all three conditions. The timing of the presentation of these tones was yoked to the recorded times of the key response during the preceding illusion condition (Cogent 2000 software; Wellcome Department of Imaging Neuroscience, London, UK). After having made the key-press response, participants were instructed to relax completely in all conditions. Analyses of the functional-imaging data were performed during the period after the participants indicated that they felt illusion in the illusion condition, and during the period after the key press in the asynchronous and incongruent conditions. It is noteworthy that, during these periods, the participants relaxed and performed no active task.

After the scanning procedures, when participants were outside the scanner, they completed the rubber-hand questionnaire describing the illusion statement ("touching own hand") (see Fig. 3D). The participants were asked to rate the average sensation across the whole experiment for each of the three stimulation conditions.

\section{Acquisition and analysis of functional-imaging data}

The functional imaging was conducted with a Siemens Allegra 3.0 T scanner (Siemens, Erlangen, Germany) to acquire gradient echo, $\mathrm{T}^{\star}$ weighted echo-planar images with blood oxygenation level-dependent contrast (BOLD) (Kwong et al., 1992; Ogawa et al., 1992) as an index of local increases in synaptic activity (Logothetis et al., 2001). The image parameters used were as follows: matrix size, $64 \times 64$; voxel size, $3 \times 3$ $\mathrm{mm}$; echo time, $40 \mathrm{~ms}$, and repetition time, $2600 \mathrm{~ms}$. A functional image volume comprised 40 contiguous horizontal slices of $2.5 \mathrm{~mm}$ thickness (with a $1.25 \mathrm{~mm}$ interslice gap), which ensured that the whole brain was within the field of view. Four experimental runs, each lasting $13 \mathrm{~min}$, were performed for each participant. For each of these runs, we collected 302 image volumes, with one volume being collected every $2.6 \mathrm{~s}$. The three stimulation conditions were repeated four times in each run in a pseudorandomized order. Each condition lasted for $42 \mathrm{~s}$. Rest conditions (20 s long) were performed before and after each stimulation condition. A high-resolution, T1-weighted structural image was also collected at the end of the experiment [using a modified driven equilibrium Fourier transform sequence (Ugurbil et al., 1993) with optimized parameters as described by Deichmann et al. (2004)].

The fMRI data were analyzed using the Statistical Parametric Mapping software (SPM2) (http://www.fil.ion.ucl.ac.uk/spm/; Wellcome Department of Imaging Neuroscience, London, UK) (Friston et al., 2004). The images were realigned to correct for head movements, 

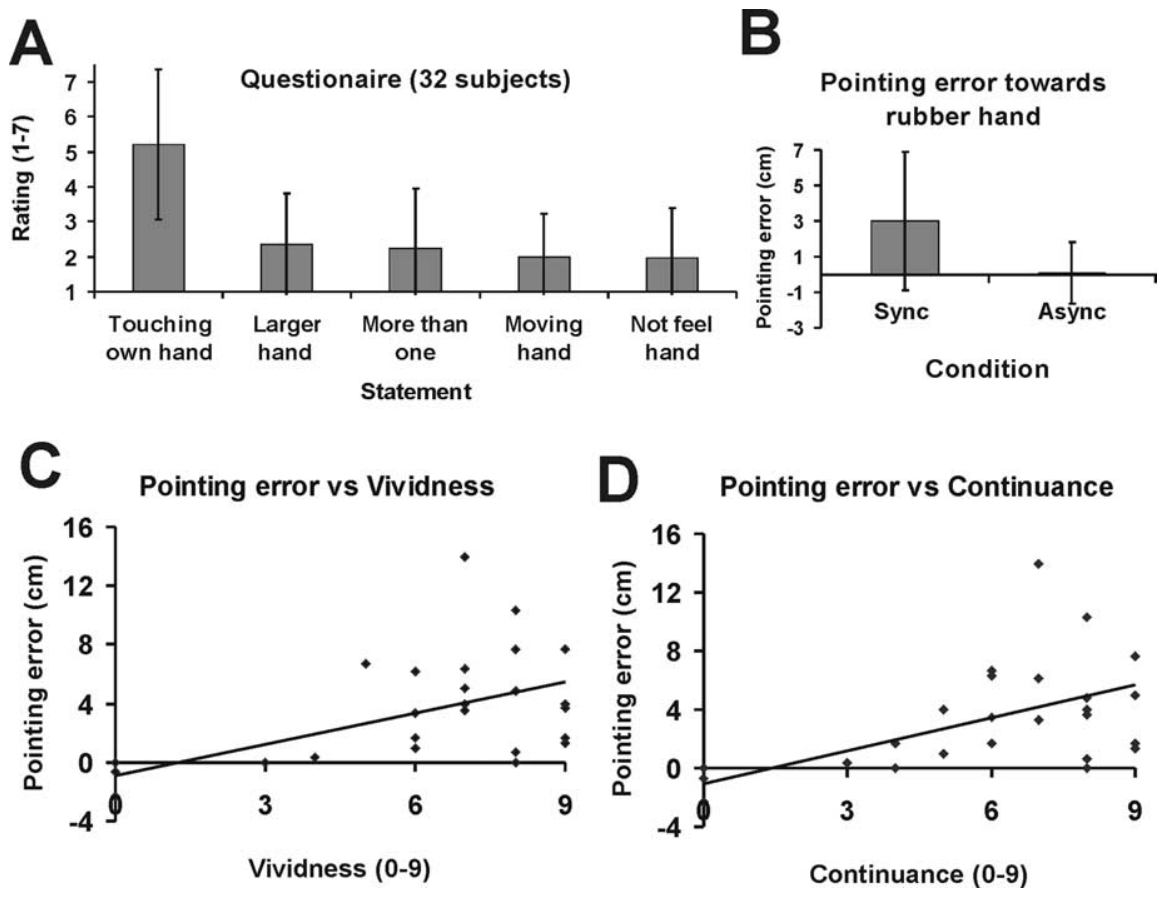

Figure 2. Testing the illusion in a group of 32 unselected participants. $A$, Results from the illusion questionnaire. The participants, on average, agreed with the statement that they felt as if they were touching their own right hand with their left index finger ("touching own hand"). The participants, on average, denied the four control statements ("larger hand"; "more than one"; "moving hand"; and "not feel hand") (see Materials and Methods for details). The differences in ratings between the illusion and the control statements were significant ( $p<0.001)$. B, Pointing errors after experiencing the illusion (sync) and after a control condition (asynchronous); the difference between ratings was significant $(p<0.001)$. C, $\boldsymbol{D}$, Pointing error against the reported vividness $(\boldsymbol{C})$ and continuance $(\boldsymbol{D})$ of the illusion of self-touch. The data have been fitted with least-squares regression lines $(\boldsymbol{C}, y=$ $\left.0.71 \times-0.94, r^{2}=0.35, p<0.001 ; \mathbf{D}, y=0.74 \times-1.05, r^{2}=0.39, p<0.001\right)$. Error bars indicate SD. Sync, Synchronous (illusion); Async, asynchronous.

coregistered with each participant's anatomical MRI, and transformed to the standard anatomical format. The functional images were spatially smoothed with a $10 \mathrm{~mm}$ full-width at half-maximum (FWHM) isotropic Gaussian kernel, and smoothed in time by a $4 \mathrm{~s}$ FWHM Gaussian kernel.

For each individual participant, we fitted a linear regression model (general linear model) to the data. Each condition was modeled with a boxcar function delayed by $4 \mathrm{~s}$ and convolved with the standard SPM2 hemodynamic response function. The periods before the key press and the key presses themselves were all modeled as conditions of no interest (block and event-related response, respectively). We defined linear contrasts in the general linear model to test our hypothesis. The results from this analysis provided the estimated BOLD signals for this contrast from each of the 15 participants (contrast images). To accommodate interparticipant variability, the contrast images from all participants were entered into a random effect group analysis (second-level analysis). One-sample one-tailed $t$ tests were used $(\mathrm{df}=14)$. We initially used the threshold of $p<0.001$, uncorrected for the statistical parametric maps to obtain cluster images. For the statistical inferences, we used the threshold of $p<$ 0.05 , corrected after a correction for the number of multiple comparisons. Because we had an a priori anatomical hypothesis that bilateral premotor cortex, left intraparietal cortex, and bilateral cerebellum would be involved (see Introduction), we used small volume corrections in these regions. Thus, we defined regions of interest using spheres of $20 \mathrm{~mm}$ radius around significant peaks taken from Ehrsson et al. (2004). In these regions, we used the threshold of $p<0.05$, corrected, and in the table and figures, we indicate all regions that survived this threshold.

To reveal activity related to the illusion after the key press, we compared the illusion condition to the two control conditions using the following contrast: (illusion - asynchronous) + (illusion - incongruent). This contrast corresponds to a main effect of illusion. Activity re- vealed by this main effect cannot be explained by the effect of synchrony alone, because touches were also synchronous in the incongruent condition.

Next, we investigated whether there was a relationship between the activity in the brain regions of interest (premotor, parietal, and cerebellum) and the strength of the illusion as rated by the subjects. Because we had quantified the vividness and continuance of the illusion in test sessions just before the scans, when the subjects lay in the same position, we could examine how the BOLD signal related to these illusion ratings. This approach is valid because we know from previous studies (Ehrsson et al., 2004), pilot experiments, and the present illusion scores obtained before and after the scans (before, $6.5 \pm 0.6$; after, $6.8 \pm 04$ ) that the rubber-hand illusion is consistent across tests within the same individual and that the main source of variance is between subjects. To obtain one value per participant that reflected the overall strength of illusion experienced during the illusion condition, we multiplied the vividness and continuance scores. This index corresponds to the integrated illusion, which should be directly related to the average fMRI signal during the illusion periods. Thus, we used this illusion index and the contrast images from the contrast illusion minus asynchronous in a linear regression analysis to correlate the strength of the illusion with the degree of activation. We focused this post hoc analysis on the areas that were more active during illusion than the controls in the main analysis and that corresponded to our a priori hypothesis (i.e., premotor cortex, parietal cortex, and cerebellum).

The search space corresponded to a $20 \mathrm{~mm}$ sphere around the relevant peaks.

The anatomical localization of the activations was related to the major sulci and gyri (Duvernoy, 1991), distinguishable on a mean MRI generated from the standardized anatomical MRIs from the 15 participants. For the cerebellum, we use the terminology of the Schmahmann atlas (Schmahmann et al., 2000).

\section{Results}

\section{Psychophysics: all participants}

The results from the illusion questionnaire showed that the participants experienced the illusion (Fig. 2A). Twenty-five participants reported that they experienced the illusion and seven participants did not. The mean rating score across all participants for "touching own hand" was $5.2(\mathrm{SD}, 2.2)$, where ratings between 5 and 7 meant affirming the presence of the illusion. Furthermore, the mean rating across all participants for the four control statements was $2.1( \pm 1.4)$, where ratings between 1 and 3 meant that the statements did not apply. The difference in ratings between the illusion statement and the control statements was significant $\left(\right.$ ANOVA, $F_{(1,31)}=27.7, p<0.001$; paired one-tailed $t$ tests, $t>$ 6.84, df $=31, p<0.001$; after correction for multiple comparisons).

In a separate session, the participants were required to point toward the index finger of their right hand after the illusion or asynchronous conditions (Fig. $2 B$ ) (data from 28 participants). The mean pointing error was $3.0 \mathrm{~cm}( \pm 3.9 \mathrm{~cm})$, which corresponded to $26 \%$ of the distance between the index fingers of the rubber hand and the real hand. No such pointing error was observed after a period of asynchronous touches $(0.1 \pm 1.7 \mathrm{~cm})$, and 


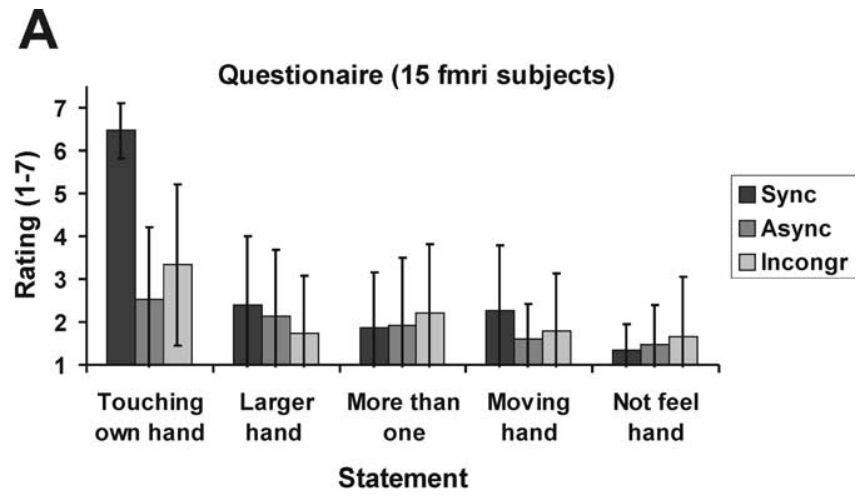

B

Vividness and Continuance
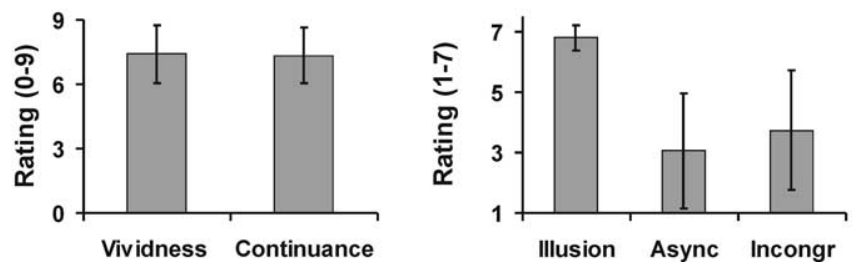

Figure 3. Testing the illusion in 15 participants selected for participation in the fMRI scanning. $A$, Results from the rubber-hand illusion questionnaire for the three stimulation conditions (see Materials and Methods). The participants, on average, reported that they felt the illusion only during the illusion condition (Sync; $p<0.001$ ), and on average they denied the four control statements. $\boldsymbol{B}$, In the illusion condition, the participants experienced a strong and persistent illusion, as revealed by the vividness and continuance ratings. $\boldsymbol{C}$, Finally, after the scans, participants reported having experienced a stronger illusion during the illusion compared with the control conditions ( $p<0.001$ ), consistent with the prescan psychophysical testing sessions. Error bars indicate SD. Sync, Synchronous (illusion); Async, asynchronous; Incongr, incongruent.

this difference between the conditions was significant (paired one-tailed $t$ test; $t=-4.87$; $\mathrm{df}=28$; $p<0.001$ ). Thus, the experience of the illusion was associated with a distortion of the felt position of the right index finger toward the location of the rubber hand. This conclusion was further strengthened by the fact that we found a significant correlation between the subjective ratings of the illusion obtained during the illusion condition and the pointing error (Fig. $2 C, D$ ). There was a significant correlation between the vividness (Fig. 2C) $\left(r^{2}=\right.$ $0.35 ; p<0.001)$ and continuance (Fig. $2 D)\left(r^{2}=0.39 ; p<\right.$ $0.001)$ and the pointing error, and between the pointing error and the questionnaire ratings of the illusion $\left(r^{2}=0.36 ; p<\right.$ 0.001) (data not shown).

\section{Psychophysics: fMRI participants}

Figure 3 summarizes the psychophysical test results of the 15 participants who took part in the fMRI experiment. We performed two analyses. First, we compared the ratings on the question concerning the illusion with the ratings on the four control questions. During the illusion, the participants were more likely to report that the rubber hand that they were touching was their own hand (repeated-measures ANOVA, $F_{(1,14)}=45.78, p<$ 0.001 ; paired one-tailed $t$ tests, $t>8.75$, $\mathrm{df}=14, p<0.001$, corrected for multiple comparisons). Second, we compared the ratings for questionnaire statements across the three conditions. During the illusion, the participants felt more strongly that the rubber hand was their own hand, compared with asynchronous (paired one-tailed $t$ test; $t=8.91 ; \mathrm{df}=14 ; p<0.001$, corrected for multiple comparisons) and incongruent conditions (paired one-tailed $t$ tests; $t=6.08 ; \mathrm{df}=14 ; p<0.001$, corrected for multiple comparisons). Figure $3 B$ displays the rated vividness and continuance of the illusion, and, as can be seen, the illusion was both vivid and persistent. Figure $3 C$ shows the results of the questionnaire in which the participants rated the illusion directly after the scan. The participants felt the illusion during illusion and not during the control conditions (paired one-tailed $t$ tests; $t>6.00 ; \mathrm{df}=14 ; p<0.001$, corrected for multiple comparisons), entirely consistent with tests performed before the scans. Finally, the fMRI participants showed a pointing error after they experienced the illusion $(4.3 \pm 2.8 \mathrm{~cm})$ but no such pointing error after a period of asynchronous touches $(0.1 \pm 1.0 \mathrm{~cm})$, and the difference between the two conditions was significant (paired onetailed $t$ test; $t=-5.85 ; \mathrm{df}=14 ; p<0.001)$.

\section{Brain imaging}

We searched for activity associated with the illusion condition [(illusion - asynchronous) + (illusion - incongruent)]. Table 1 and Figure 4 show that bilateral activity was found in the ventral premotor region (PMv), intraparietal cortex, and cerebellum $(p<0.05$, corrected). In the premotor region, the activity was located in the right inferior part of the precentral sulcus and the left inferior part of the precentral sulcus ( $p<0.05$, small volume corrected) (Table 1). It is not possible to determine whether the peaks in the inferior precentral sulcus lay within premotor area 6 or the posterior part of area 44 . The posterior bank of the inferior part of the precentral sulcus corresponds to area 6 , and the anterior bank to the posterior part of area 44 . The activation of the right $\mathrm{PMv}$ was more extensive than on the left side and extended dorsally into the dorsal premotor cortex (PMd). Activation peaks were also detected in the left frontal operculum $(p<0.05$, corrected), which is a region that is anatomically adjacent to the premotor cortex. In the right frontal operculum, a response was observed that did not reach significance after correction for multiple comparisons $(x=57 ; y=18 ; z=3$; $t$ value, $4.72 ; p<0.001$, uncorrected). In the parietal cortex, a significant peak of activation was located in the left cortices lining the intraparietal sulcus ( $p<0.05$, corrected). This activity was probably bilateral, because we observed activity in the right intraparietal cortex at the corresponding site $(p<0.001$, uncorrected). The right-sided activation was not significant after correction for multiple comparisons, because it was not part of our a priori hypothesis. In the cerebellum, several foci of activity were observed in the lateral hemispheres bilaterally and in the medial cerebellum $(p<0.05$, corrected).

The analysis above compared the activity in the illusion condition with the average activity in the two control conditions. To exclude the possibility that our results merely related to a large difference between the illusion condition and one of the controls, we conducted a conjunction analysis [(illusion - asynchronous) and (illusion - incongruent)]. This analysis identifies regions that show greater activity in the illusion condition than in each of the two controls. We found significant $(p<0.05$, corrected) activation bilaterally in the ventral premotor cortex, in the left intraparietal cortex, and in the medial and right cerebellum at the same sites as detected in the main analysis described above (in the left cerebellum, activity was seen at $p=0.001$, uncorrected). Thus, the activity in these areas was greatest in the illusion condition (Fig. 4).

This is further illustrated in Figure 5, where we show the parameter estimates for the three experimental conditions for the bilateral premotor cortex, the left intraparietal cortex, and the 
Table 1. Illusion-related activity (main effect of illusion)

\begin{tabular}{|c|c|c|c|c|}
\hline \multirow[b]{2}{*}{ Anatomical region } & \multicolumn{4}{|c|}{$\begin{array}{l}\text { Main peaks } \\
p<0.05 \text { corrected }^{a, b}\end{array}$} \\
\hline & $x$ & $y$ & $z$ & Peak z value \\
\hline \multicolumn{5}{|l|}{ Frontal areas } \\
\hline L. frontal operculum & -54 & 12 & 3 & 4.46 \\
\hline L. precentral sulcus (PMv) & -60 & 9 & 9 & 3.84 \\
\hline R. precentral gyrus (PMd) & 45 & -6 & 57 & 3.85 \\
\hline R. precentral sulcus (PMv) & 48 & 9 & 30 & 3.61 \\
\hline \multicolumn{5}{|l|}{ Parietal areas } \\
\hline L. intraparietal sulcus & -39 & -57 & 51 & 4.22 \\
\hline R. intraparietal sulcus & 36 & -57 & 60 & $4.25^{c}$ \\
\hline \multicolumn{5}{|l|}{ Cerebellum } \\
\hline L. lat. cerebellum (lobule VI/crus I) & -33 & -72 & -24 & 4.16 \\
\hline L. lat. cerebellum (crus I) & -15 & -81 & -27 & 3.37 \\
\hline L. medial cerebellum (crus II) & -6 & -81 & -30 & 3.54 \\
\hline R. lat. cerebellum (lobule VI/crus I) & 36 & -75 & -24 & 4.29 \\
\hline
\end{tabular}

L., Left; R., right; lat., lateral.

${ }^{a}$ Small volume corrections based on an a priori hypothesis.

${ }^{b} 0$ nly the most significant peaks from each region are reported.

'This peak was not significant after correction for multiple comparisons ( $p<0.001$ uncorrected; $p>0.05$ corrected), but we report it to illustrate the bilateral pattern of activity in the intraparietal cortex.

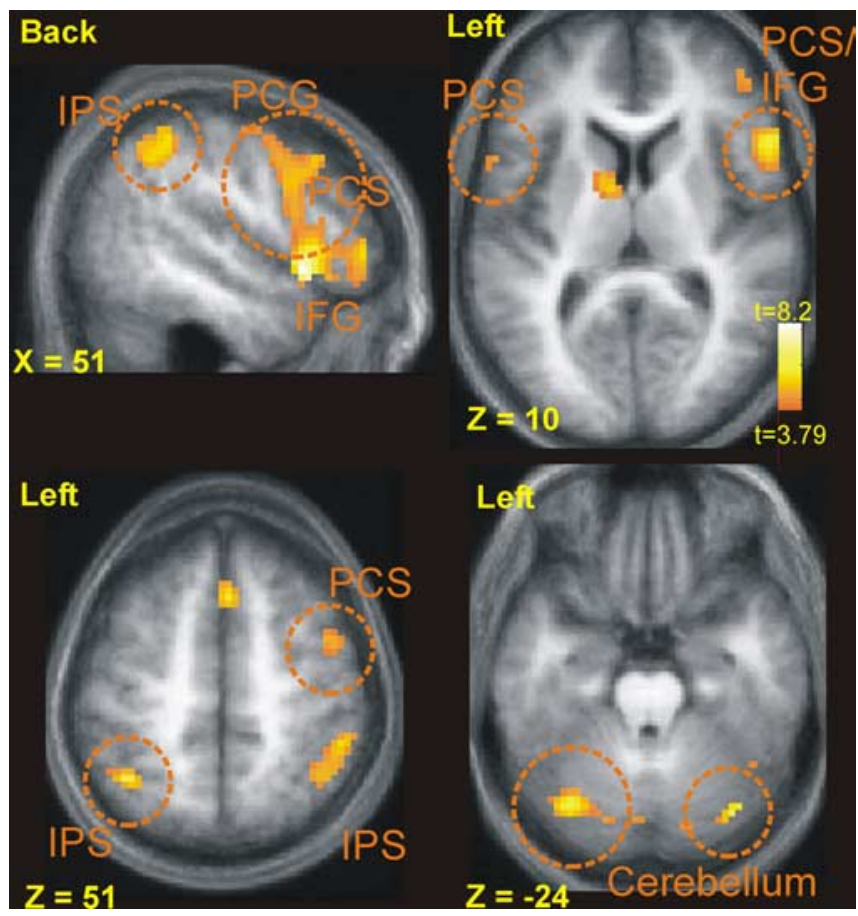

Figure 4. Activity in the premotor cortex (top panels), intraparietal cortex (left lower), and the cerebellum (right lower) that reflected the illusion of touching one's own hand (self-touch). The yellow/red activations correspond to the statistical parametric map of the contrast [(illusion - asynchronous) + (illusion - incongruent)] ( $p<0.001$, uncorrected). The significant activations are indicated by orange circles ( $p<0.05$, corrected). This activation map is superimposed on the mean high-resolution anatomical MRI of the 15 participants on which the major sulci are visible. The right hemisphere is shown to the right. The coordinates in standard space are indicated. PCG, Precentral gyrus; PCS, precentral sulcus; IPS, intraparietal sulcus; IFG, inferior frontal gyrus.

bilateral cerebellum. In these plots, the baseline is given by the rest condition. It will be seen that, in all cases, the activity in the illusion condition was greater than the activity in either the asynchronous or incongruent conditions.

We further investigated whether the activity in these areas was related to the strength of the illusion as rated by the participants before the scan (Fig. 6). The participants who reported the stron- gest illusion during the illusion condition also showed the strongest BOLD signal in the left ventral premotor cortex (left precentral sulcus; $x=-60 ; y=6 ; z=30 ; r^{2}=$ 0.32 ; $p<0.01)$ and the right ventral premotor cortex (right precentral sulcus; $x=$ $\left.54 ; y=6 ; z=33 ; r^{2}=0.20 ; p<0.05\right)$. These peaks were located in similar regions of the inferior ramus of the precentral sulcus as the peaks detected in the main analysis described above. Also, the clusters of these activations overlapped with the clusters of active voxels detected in the main analysis $(p<0.05$, uncorrected). Also, we found a positive correlation between neural activity and illusion strength in the left lateral cerebellum (lobule VI; $x=-15 ; y=-68 ; z=-18 ; r^{2}=$ $0.62 ; p<0.001)$ and the right lateral cerebellum (lobule VI; $x=33, y=-60, z=$ $\left.-24 ; r^{2}=0.37 ; p<0.01\right)$. No significant positive correlations were observed between the strength of the illusion and activity in bilateral parietal cortex, insula, thalamus, medial wall of the frontal lobes, or in the prefrontal cortex ( $p>0.01$, uncorrected).

\section{Discussion}

There were two main findings. First, we described a new version of the rubber-hand illusion, in which blindfolded persons felt that a rubber hand they touched was their own hand. This shows that the rubber-hand illusion is not simply generated by the dominance of vision over somesthesis, but that temporally correlated and matching tactile and proprioceptive signals from two body parts is sufficient to change the feeling of ownership of a touched rubber limb. Second, we showed that bilateral activity in the ventral premotor cortex and cerebellum was greater during the illusion than during the control conditions and that there was a linear relationship between the degree of activation in these areas and the participant's ratings of the illusion. This supports our argument in our previous study (Ehrsson et al., 2004) that the premotor activity reflected the integration of correlated visual, tactile, and proprioceptive signals from the hand, and not the visual representation of an object in peripersonal space (Botvinick, 2004). Together, these findings provide strong support for the hypothesis that the feeling of body ownership is mediated by the detection of correlated multisensory signals and that the premotor cortex and the cerebellum play important roles in this process.

The psychophysical experiment demonstrated that the illusion depended on the temporal synchrony of the sensory signals from the two hands, and the similarity of the shape and texture of the touched objects and the participant's own hand. Furthermore, the illusion was associated with the recalibration of position sense of the touched (right) hand (the left hand was not tested) (Fig. $2 B-D$ ). The reported subjective experience of the illusion, the time course of its onset, and the recalibration of position sense suggest that the present somatic rubber-hand illusion and the visual rubber-hand illusion (Botvinick and Cohen, 1998; Ehrsson et al., 2004) share common underlying mechanisms. That synchronous tactile stimuli on two body parts can cause illusory distortions in size, shape, and location of body parts has been described before (Craske et al., 1984; Ramachandran and Hirstein, 1998) [for example, in the "phantom nose 
illusion" described by Ramachandran and Hirstein (1998)]. However, in these previous studies, the issue of body ownership was not investigated.

That the ventral premotor cortex contributes to self-attribution of body parts is consistent with the well established role of this area in multisensory integration. The ventral premotor cortex receives visual, tactile, and proprioceptive input (Graziano and Gross, 1998; Rizzolatti et al., 2002), and it is anatomically connected with higher-order somatosensory areas such as the secondary somatosensory cortex and the inferior parietal cortex (area 7b) (Godschalk et al., 1984; Matelli et al., 1986; Rizzolatti et al., 1998). In the monkey brain, premotor neurons discharge when the hand is touched or when a visual stimulus is presented near the hand (Rizzolatti et al., 1981a,b; Gentilucci et al., 1983; Fogassi et al., 1992; Graziano et al., 1994), and premotor neurons also represent the position of the hand (Graziano, 1999; Lloyd et al., 2003). The receptive fields of the visually responsive cells are "anchored" to the hand, so that when the position of the hand changes, the receptive fields follow the hand (i.e., these cells represent the space near the hand in a bodycentered reference frame) (Graziano et al., 1994, 1997a). In the present experiment, activity in the ventral premotor cortex cannot reflect a visual representation of an object near the hand in peripersonal space, because the participants were blindfolded. Furthermore, the activity cannot reflect a nonvisual representation of an object near the hand (Graziano et al., 1997b), because in all conditions, the participant's right hand was touched by the experimenter's finger. Furthermore, the elicitation of the rubber-hand illusion does not require that an object is touching or approaching the hand. Pilot experiments showed that the illusion can easily be elicited using passive movements and, in some participants, spontaneously by just looking at the static fake hand for a while (Tastevin, 1937; Pavani et al., 2000). Thus, the premotor activity probably reflects the detection and integration of congruent tactile, proprioceptive, and visual signals from the hands, rather than the representation of an external object in peripersonal space. Premotor activity reflecting this type of multisensory integration could be related to the feeling of body ownership. Evidence for this is the observed correlation between premotor activity and the participant's ratings of the strength of the illusion.

The premotor activation peaks in the present study lay in the bilateral inferior ramus of the precentral sulcus, as they did in our previous study of the visual rubber-hand illusion (Ehrsson et al., 2004), although the exact coordinates in standard space differed somewhat. However, it should be made clear that we cannot determine whether exactly the same neuronal populations were active in the two studies. The statistical images have limited resolution, and different groups of subjects were used in the two studies. The activation of the right ventral premotor region was more extensive than on the left, and extended more dorsally with a peak in the PMd. This difference could reflect the dominance of the right hemisphere for body perception (Carpenter et al., 1995; Meador et al., 2000; Naito et al., 2005).

It now seems increasingly clear that the lateral cerebellum is involved in the rubber-hand illusion. As described above, we found previously a significant correlation between activity in the lateral cerebellum and the strength of the visual rubberhand illusion, as well as cerebellar responses reflecting the synchrony of the brushstrokes and the orientation of the rubber arm (Ehrsson et al., 2004). In the present study, the significant activations associated with the illusion condition were located in lobules VI and crus I of the cerebellar hemispheres. Likewise, the degree of activity in bilateral lobules VI was related to the strength of the illusion as reported by the participants. These sections of the cerebellum receive inputs from the premotor and parietal cortices via the pontine nuclei, and then send information back to these cortical areas via the dentate nuclei and the ventrolateral thalamus (Schmahmann and Pandya, 1997; Clower et al., 2001, 2005; Dum et al., 2002; Dum and Strick, 2003). Thus, the cerebellar hemispheres have the capacity to integrate tactile, proprioceptive, and visual repre- 


\section{L. precentral s. (-60 630$)$}

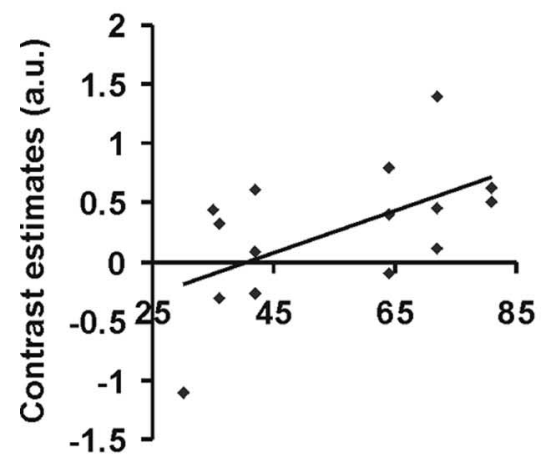

Strength of illusion (0-89)

L. cerebellum (-15 -68 -18)

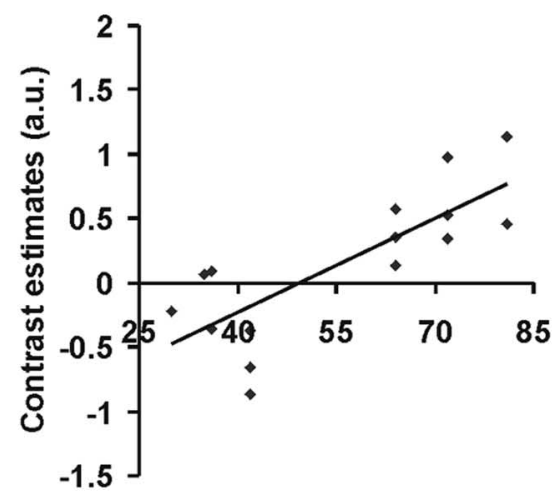

Strength of illusion (0-89)
R. inf. precentral s. (54 6 33)

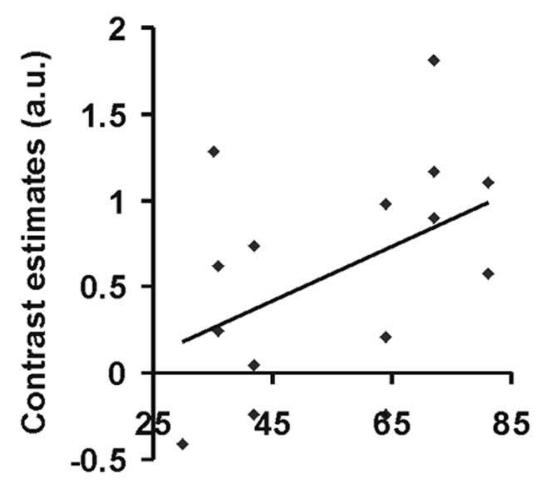

Strength of illusion (0-89)

R. cerebellum (33 -60 -24)

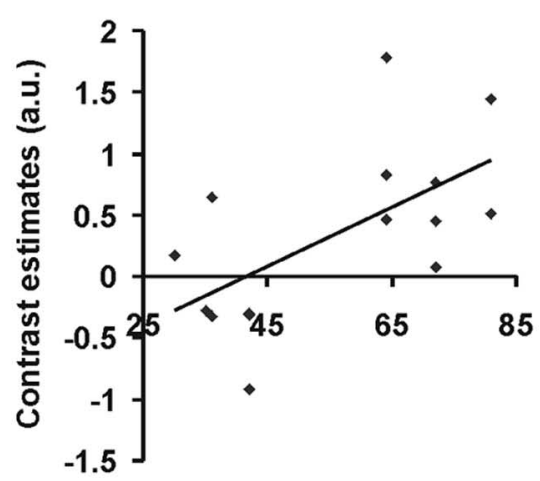

Strength of illusion $(0-89)$

Figure 6. Relationship between activity in premotor (precentral, top panels) and cerebellar (bottom panels) cortices, and the reported strength of the illusion (linear regression analysis). The $y$-axis shows the contrast estimates for the contrast (illusion asynchronous). The $x$-axis shows the illusion index (the product of the vividness and continuance ratings). The data have been fitted with least-squares regression lines (left precentral sulcus, $y=0.018 \times-0.726, r^{2}=0.32, p<0.01$; right precentral sulcus, $y=0.016 \times-0.877, r^{2}=0.20, p<0.05$; left cerebellum, $y=0.025 \times-1.21, r^{2}=0.62, p<0.001$; right cerebellum, $y=0.024 \times-0.99, r^{2}=0.37, p<0.01$ ). The peaks were located close to those detected in the main analysis. a.u., Arbitrary unit; L., left; R., right; inf., inferior; s., sulcus.

sentations of the body, consistent with the view that the cerebellum has a role in the analysis of sensory information (Stein and Glickstein, 1992; Gao et al., 1996; Jueptner et al., 1997; Naito et al., 2002; Liu et al., 2003). Furthermore, neurophysiological, lesion, and neuroimaging data suggest that the cerebellum is particularly important for the analysis of the timing of sensory and motor signals (Perrett et al., 1993; Ivry, 1996; Blakemore et al., 2001). However, in the present experiment, the cerebellar activity was greater in the illusion condition than in the incongruent condition, which also included synchronous stimulation. Thus, it cannot be accounted for by the effect of synchrony as such. This is not to say that the cerebellum is not involved in the detection of synchrony (Bushara et al., 2003; Ehrsson et al., 2004), but that the cerebellum is particularly involved in the detection of temporally correlated signals when the tactile information from two body parts is matched.

It has also been proposed that cerebellar mechanisms are involved in the distinction between self-produced sensory signals and externally produced stimuli by detecting mismatches between the predicted sensory consequences of the voluntary movements and the actual sensory feedback (Miall et al., 1993; Wolpert et al., 1995; Blakemore et al., 1998, 2001; Wolpert and Ghahramani, 2000). Empirical evidence for this is that cerebellar activity increases during externally produced touch (Blakemore et al., 1998), and there is a positive correlation between cerebellar activity and the asynchrony between the movements of one hand and tactile stimulation on the other (Blakemore et al., 2001). However, this mechanism can probably not explain the present results. We found greater activity in the illusion than asynchrony, and a positive correlation with the reported strength of the illusion, and recall that this illusion was reduced by asynchrony.

The posterior parietal cortex is probably intimately involved in the process of multisensory integration during the illusion. As in our previous study (Ehrsson et al., 2004), activity was detected in the cortices lining the intraparietal cortex. This region is connected to visual, somatosensory, and premotor areas (Jones et al., 1978; Johnson et al., 1996; Rizzolatti et al., 1998), and neurons in this region integrate visual, tactile, and proprioceptive information from the hand (Sakata et al., 1973; Colby and Duhamel, 1991; Iriki et al., 1996; Graziano et al., 2000; Graziano and Botvinick, 2001). In monkey area 5, many somatosensory cells have bilateral receptive fields, that is, they receive somatic information from both of the upper limbs (Iwamura et al., 2002). Thus, the intraparietal activity we detected probably reflects neuronal populations that integrate tactile and proprioceptive representations from the two hands and that work together with the premotor cortex and the cerebellum. However, it is still somewhat unclear whether the activity in the intraparietal cortex reflects the feelings of ownership per se, because we did not detect a significant correlation between the illusion-related activity and the subjective ratings of the illusion, even when we lowered the threshold for significance ( $p>0.01$, uncorrected).

In conclusion, the present findings associate activity in premotor, intraparietal, and cerebellar regions with the feeling of body ownership when touching one's own limbs. This, together with the results from our previous experiment (Ehrsson et al., 2004), supports the hypothesis that the detection of correlated multisensory signals by these regions is the mechanism for body ownership.

\section{References}

Armel KC, Ramachandran VS (2003) Projecting sensations to external objects: evidence from skin conductance response. Proc R Soc Lond B Biol Sci 270:1499-1506.

Bahrick LE, Watson JS (1985) Detection of intermodal proprioceptivevisual contingency as a potential basis of self-perception in infancy. Dev Psychol 21:963-973. 
Blakemore SJ, Wolpert DM, Frith CD (1998) Central cancellation of selfproduced tickle sensation. Nat Neurosci 1:635-640.

Blakemore SJ, Frith CD, Wolpert DM (2001) The cerebellum is involved in predicting the sensory consequences of action. NeuroReport 12:1879-1884.

Botvinick M (2004) Neuroscience. Probing the neural basis of body ownership. Science 305:782-783.

Botvinick M, Cohen J (1998) Rubber hands "feel" touch that eyes see. Nature 391:756.

Bushara KO, Hanakawa T, Immisch I, Toma K, Kansaku K, Hallett M (2003) Neural correlates of cross-modal binding. Nat Neurosci 6:190-195.

Carpenter K, Berti A, Oxbury S, Molyneux AJ, Bisiach E, Oxbury JM (1995) Awareness of and memory for arm weakness during intracarotid sodium amytal testing. Brain 118:243-251.

Clower DM, West RA, Lynch JC, Strick PL (2001) The inferior parietal lobule is the target of output from the superior colliculus, hippocampus, and cerebellum. J Neurosci 21:6283-6291.

Clower DM, Dum RP, Strick PL (2005) Basal ganglia and cerebellar inputs to "AIP." Cereb Cortex 7:913-920.

Colby CL, Duhamel JR (1991) Heterogeneity of extrastriate visual areas and multiple parietal areas in the macaque monkey. Neuropsychologia 29:517-537.

Craske B, Kenny FT, Keith D (1984) Modifying an underlying component of perceived arm length: adaptation of tactile location induced by spatial discordance. J Exp Psychol Hum Percept Perform 10:307-317.

Deichmann R, Schwarzbauer C, Turner R (2004) Optimisation of the 3D MDEFT sequence for anatomical brain imaging: technical implications at 1.5 and 3 T. NeuroImage 21:757-767.

Dum RP, Strick PL (2003) An unfolded map of the cerebellar dentate nucleus and its projections to the cerebral cortex. J Neurophysiol 89:634-639.

Dum RP, Li C, Strick PL (2002) Motor and nonmotor domains in the monkey dentate. Ann NY Acad Sci 978:289-301.

Duvernoy HM (1991) The human brain: surface, blood supply, and threedimensional sectional anatomy. New York: Springer.

Ehrsson HH, Spence C, Passingham RE (2004) That's my hand! Activity in premotor cortex reflects feeling of ownership of a limb. Science 305:875-877.

Fogassi L, Gallese V, di Pellegrino G, Fadiga L, Gentilucci M, Luppino G, Matelli M, Pedotti A, Rizzolatti G (1992) Space coding by premotor cortex. Exp Brain Res 89:686-690.

Friston K, Ashburner J, Penny W, Kiebel S, Holmes A, Poline JB, Kherif F, Glaser JB, Henson R, Mechelli A, Turner R, Price C, Brett M, Worsley K, Nichols T, Buchüel C, Harrison L (2004) Part two. Imaging neuroscience: theory and analysis. In: Human brain function (Frackowiak RS, Friston K, Frith CD, Dolan RJ, Price CJ, Zeki S, Ashburner J, Penny W, eds), pp 599-968. San Diego: Elsevier, Academic.

Gao JH, Parsons LM, Bower JM, Xiong J, Li J, Fox PT (1996) Cerebellum implicated in sensory acquisition and discrimination rather than motor control. Science 272:545-547.

Gentilucci M, Scandolara C, Pigarev IN, Rizzolatti G (1983) Visual responses in the postarcuate cortex (area 6) of the monkey that are independent of eye position. Exp Brain Res 50:464-468.

Godschalk M, Lemon RN, Kuypers HG, Ronday HK (1984) Cortical afferents and efferents of monkey postarcuate area: an anatomical and electrophysiological study. Exp Brain Res 56:410-424.

Graziano MS (1999) Where is my arm? The relative role of vision and proprioception in the neuronal representation of limb position. Proc Natl Acad Sci USA 96:10418-10421.

Graziano MS, Botvinick M (2001) How the brain represents the body: insights from neurophysiology and psychology. In: Common mechanisms in perception and action, attention and performance, Vol XIX (Prinz W, Hommel B, eds), pp 136-157. Oxford: Oxford UP.

Graziano MS, Gross CG (1998) Spatial maps for the control of movement. Curr Opin Neurobiol 8:195-201.

Graziano MS, Yap GS, Gross CG (1994) Coding of visual space by premotor neurons. Science 266:1054-1057.

Graziano MS, Hu XT, Gross CG (1997a) Visuospatial properties of ventral premotor cortex. J Neurophysiol 77:2268-2292.

Graziano MS, Hu XT, Gross CG (1997b) Coding the locations of objects in the dark. Science 277:239-241.
Graziano MS, Cooke DF, Taylor CS (2000) Coding the location of the arm by sight. Science 290:1782-1786.

Iriki A, Tanaka M, Iwamura Y (1996) Coding of modified body schema during tool use by macaque postcentral neurones. NeuroReport 7:2325-2330.

Ivry RB (1996) The representation of temporal information in perception and motor control. Curr Opin Neurobiol 6:851-857.

Iwamura Y, Tanaka M, Iriki A, Taoka M, Toda T (2002) Processing of tactile and kinesthetic signals from bilateral sides of the body in the postcentral gyrus of awake monkeys. Behav Brain Res 135:185-190.

Johnson PB, Ferraina S, Bianchi L, Caminiti R (1996) Cortical networks for visual reaching: physiological and anatomical organization of frontal and parietal lobe arm regions. Cereb Cortex 6:102-119.

Jones EG, Coulter JD, Hendry SH (1978) Intracortical connectivity of architectonic fields in the somatic sensory, motor and parietal cortex of monkeys. J Comp Neurol 181:291-347.

Jueptner M, Ottinger S, Fellows SJ, Adamschewski J, Flerich L, Muller SP, Diener HC, Thilmann AF, Weiller C (1997) The relevance of sensory input for the cerebellar control of movements. NeuroImage 5:41-48.

Kwong KK, Belliveau JW, Chesler DA, Goldberg IE, Weisskoff RM, Poncelet BP, Kennedy DN, Hoppel BE, Cohen MS, Turner R (1992) Dynamic magnetic resonance imaging of human brain activity during primary sensory stimulation. Proc Natl Acad Sci USA 89:5675-5679.

Liu X, Robertson E, Miall RC (2003) Neuronal activity related to the visual representation of arm movements in the lateral cerebellar cortex. J Neurophysiol 89:1223-1237.

Lloyd DM, Shore DI, Spence C, Calvert GA (2003) Multisensory representation of limb position in human premotor cortex. Nat Neurosci 6:17-18.

Logothetis NK, Pauls J, Augath M, Trinath T, Oeltermann A (2001) Neurophysiological investigation of the basis of the fMRI signal. Nature 412:150-157.

Matelli M, Camarda R, Glickstein M, Rizzolatti G (1986) Afferent and efferent projections of the inferior area 6 in the macaque monkey. J Comp Neurol 251:281-298.

Meador KJ, Loring DW, Feinberg TE, Lee GP, Nichols ME (2000) Anosognosia and asomatognosia during intracarotid amobarbital inactivation. Neurology 55:816-820.

Miall RC, Weir DJ, Wolpert DM, Stein JF (1993) Is the cerebellum a Smith predictor? J Mot Behav 25:203-216.

Mitchell RW (1997) A comparison of the self-awareness and kinestheticvisual matching theories of self-recognition: autistic children and others. Ann NY Acad Sci 818:38-62.

Naito E, Roland PE, Ehrsson HH (2002) I feel my hand moving: a new role of the primary motor cortex in somatic perception of limb movement. Neuron 36:979-988.

Naito E, Roland PE, Grefkes C, Choi HJ, Eickhoff S, Geyer S, Zilles K, Ehrsson $\mathrm{HH}$ (2005) Dominance of the right hemisphere and role of area 2 in human kinesthesia. J Neurophysiol 93:1020-1034.

Ogawa S, Tank DW, Menon R, Ellermann JM, Kim SG, Merkle H, Ugurbil K (1992) Intrinsic signal changes accompanying sensory stimulation: functional brain mapping with magnetic resonance imaging. Proc Natl Acad Sci USA 89:5951-5955.

Pavani F, Spence C, Driver J (2000) Visual capture of touch: out-of-thebody experiences with rubber gloves. Psychol Sci 11:353-359.

Perrett SP, Ruiz BP, Mauk MD (1993) Cerebellar cortex lesions disrupt learning-dependent timing of conditioned eyelid responses. J Neurosci 13:1708-1718.

Ramachandran VS, Hirstein W (1998) The perception of phantom limbs. The D. O. Hebb Lecture. Brain 121:1603-1630.

Rizzolatti G, Scandolara C, Matelli M, Gentilucci M (1981a) Afferent properties of periarcuate neurons in macaque monkeys. I. Somatosensory responses. Behav Brain Res 2:125-146.

Rizzolatti G, Scandolara C, Matelli M, Gentilucci M (1981b) Afferent properties of periarcuate neurons in macaque monkeys. II. Visual responses. Behav Brain Res 2:147-163.

Rizzolatti G, Luppino G, Matelli M (1998) The organization of the cortical motor system: new concepts. Electroencephalogr Clin Neurophysiol 106:283-296.

Rizzolatti G, Fogassi L, Gallese V (2002) Motor and cognitive functions of the ventral premotor cortex. Curr Opin Neurobiol 12:149-154.

Rochat P (1998) Self-perception and action in infancy. Exp Brain Res 123:102-109. 
Sakata H, Takaoka Y, Kawarasaki A, Shibutani H (1973) Somatosensory properties of neurons in the superior parietal cortex (area 5) of the rhesus monkey. Brain Res 64:85-102.

Schmahmann JD, Pandya DN (1997) The cerebrocerebellar system. Int Rev Neurobiol 41:31-60.

Schmahmann JD, Doyon J, Toga AW, Petrides M, Evans AC (2000) MRI atlas of the human cerebellum. San Diego: Academic.

Stein JF, Glickstein M (1992) Role of the cerebellum in visual guidance of movement. Physiol Rev 72:967-1017.

Tastevin J (1937) En partant de l'e expérience d'Aristote: les déplacements artificiels des parties du corps ne sont pas suivis par le sentiment de ces parties ni pas les sensations qu'on peut y produire. L'Encephale 32:57-84, 140-158.
Tsakiris M, Haggard P (2005) The rubber hand illusion revisited: visuotactile integration and self-attribution. J Exp Psychol Hum Percept Perform 31:80-91.

Ugurbil K, Garwood M, Ellermann J, Hendrich K, Hinke R, Hu X, Kim SG, Menon R, Merkle H, Ogawa S, Salmi R (1993) Imaging at high magnetic fields: initial experiences at 4 T. Magn Reson Q 9:259-277.

van den Bos E, Jeannerod M (2002) Sense of body and sense of action both contribute to self-recognition. Cognition 85:177-187.

Wolpert DM, Ghahramani Z (2000) Computational principles of movement neuroscience. Nat Neurosci 3 [Suppl]:1212-1217.

Wolpert DM, Ghahramani Z, Jordan MI (1995) An internal model for sensorimotor integration. Science 269:1880-1882. 